\title{
Diffuse Large B-Cell Lymphoma Associated with Chronic Inflammation
}

National Cancer Institute

\section{Source}

National Cancer Institute. Diffuse Large B-Cell Lymphoma Associated with Chronic

Inflammation. NCl Thesaurus. Code C80289.

A diffuse large B-cell lymphoma arising in body cavities or narrow spaces of long standing chronic inflammation. The classic example is the pyothorax-associated lymphoma that arises in the pleural cavity of patients with a history of long standing pyothorax. 